\title{
Activities Suggestion Based on Emotions in AAL Environments
}

\author{
Angelo Costa ${ }^{\mathrm{a}, *}$, Jaime Andres Rincon ${ }^{\mathrm{b}, *}$, Carlos $\operatorname{Carrascos}^{\mathrm{b}}{ }$, Paulo Novais ${ }^{\mathrm{a}}$, Vicente \\ Julian $^{\mathrm{b}}$ \\ ${ }^{a}$ ISLab / ALGORITMI Center - University of Minho, Portugal \\ ${ }^{b}$ Departamento de Sistemas Informáticos y Computación (DSIC), Universitat Politécnica de Valéncia, Spain
}

\begin{abstract}
The elderly population is increasing and the response of the society was to provide them with services directed to them to cope with their needs. One of the oldest solutions is the retirement home, providing housing and permanent assistance for the elderly. Furthermore, most of the retirement homes are inhabited by multiple elderly people, thus creating a community of people who are somewhat related in age and medical issues. The Ambient Assisted Living (AAL) area tries to solve some of the elderly issues by producing technological products, some of them dedicated to elderly homes. One of the identified problem is that elderly people are sometimes discontent about the activities that consume most of their day promoted by the retirement home social workers. The work presented in this paper attempts to improve how these activities are scheduled taking into account the elderlies' emotional response to these activities. The aim is to maximize the group happiness by promoting the activities the group likes, minding if they are bored due to activities repetition. In this sense, this paper presents an extension of the Cognitive Life Assistant platform incorporating a social emotional model. The proposed system has been modelled as a free time activity manager which is in charge of suggesting activities to the social workers.
\end{abstract}

Keywords: Emotional Agents, Ambient Intelligence, Multi-agent Systems, Agent-based Simulation, Ambient Assisted Living

\section{Introduction}

Elderly population suffer from complicated medical conditions and require much attention from an array of people, like doctors, nurses, family and caregivers, among others. Although currently is very difficult to provide medical assistance to all of them, 5 efforts are being made to increase the number of services available to them. The assisted people resort to one or more of the following support systems: home health agencies,

\footnotetext{
* Corresponding author

Email addresses: acosta@di.uminho.pt (Angelo Costa), jrincon@dsic.upv.es (Jaime Andres Rincon), carrasco@dsic.upv.es (Carlos Carrascosa), pjon@di.uminho.pt (Paulo Novais), vinglada@dsic.upv.es (Vicente Julian)
} 
nursing homes, hospices, residential care communities and adult day service centres. For instance, in the USA annually 8357100 people receive one type of these support systems, being the home health agencies the most used service[1]. Also, the current number of people that need long-term care are about 6.3 million [2], with tendency to increase sharply in the next years. This long-term care is designed to help people with mild to severe cognitive or physical disabilities that affects $68 \%$ of people aged 65 and older[3].

In 2050 it is expected the doubling of people receiving long-term care in relation

15 to the year 2000 figures, meaning that at least 27 million people are expected to be receiving assistance and some experts project even higher numbers due to the current growth of population [4]. The issue with the presented values is that social security and governments have said that they lack the funds to support everyone, even if there are services available to everyone of them, which turns into a heavy monetary investment to 20 a person with prices ascending from 12000 Euros annually.

Thus, they have to be cared and assisted and that usually is attributed to the family, informal caregivers. The issue with resorting to them is that they are usually time constricted and may be unable to care for a person with severe disabilities. This results in severe complications both to the elderly as well as the family or friends, resulting

25 in a enormous strain to them, physically and more importantly psychologically [5]. For instance, in Portugal the latest surveys show that there are more than 49 thousand bedridden people at their family houses without access to proper medical assistance; and that there are 110355 people dependent of others to perform their activities of daily life (ADL)[6].

30 Another choice is the nursing home care, which usually welcomes people with 75 years and older or with less age if they present health problems[7]. These places and services provide constant attention and appropriate medical services to its users. Furthermore, the nursing home facilities are equipped with technological resources or can be easily upgraded to receive new devices if it is necessary. Moreover, in light 35 of the technological advances the nursing homes have been implementing Ambient Intelligence (AmI) systems aiming to improve its users everyday life [8, 9, 10].

The AmI aimed to revolutionize the way that the home environments (and environments in general) were managed by introducing perception and action features into common household devices, all of this in beneficence of the people living under those

40 environments. The information captured by the sensors systems available can be used to develop a context state, where more intricate considerations about the environment can be achieved opposed to a simpler reaction system that only responds directly to each sensor data.

Humans are complex so the environments that surround them are too. Technological 45 systems have a hard time accompanying this complexity, and while there are significant developments, considering every variable of the real world is still very much impossible. The best effort of the available technology is to provide the environments with sensors and computing power able to consider direct human interaction, alas decision processes. Decisions should be the outcome of the composite status of the perceived environment, ${ }_{50}$ where one factor (or sensor) is more important than the other, and the response to the desires of the people who inhabit the environment is paramount to the utility functions of the system. One of the AmI goals is to consider, above all, the environment users 
preferences [11, 12].

The advances of the AmI are already considerable and growing, due to the interest

55 of the scientific community and the private sector [13]. For instance, there are already devices available to be bought of the shelf, able to be integrated into an AmI platform. The impulse of the governments and even the European Union through special funding to agile the active ageing and to promote solutions that should be accomplished in the near future to comply with the crescent number of elderly people that the statistics present.

60 One of the main issues that nowadays most of the available projects and products have is the lack of re-usage and interoperability, meaning that the features that they provide cannot be extended and the devices are certainly unable to communicate if they are from different brands. It is pressing the need of integration in AmI projects, which some projects are now addressing but the results are new and without much testing [14].

Not only are there issues in terms of the architecture, but in the concept too. Only very recently there were projects that used real input from the users to conceive the project, while most of the development has taken little consideration the opinion of the people that will be using the final product. This has lead to a poor adoption of the project's resulting products and to maintain the general distrust on these type of projects.

One of the proposed solutions is making questionnaires to the target users about their needs and wants, producing a dataset of the general features that are needed by the general target population [15, 16].

One of the unexpected outcome was the inclusion of emotions and the psychological state at the same level as physical problems by the people questioned. Therefore, we

75 must assume that comfort is not only provided by the ailment of physical conditionings but also by the psychological state, as elder depression is a serious state that affects a large number of persons and has the ability to affect the physical state[17]. It is then imperative that the AmI projects develop means to access and improve the psychological state of their users.

Taking into account all of these aspects, this paper presents the Cognitive Life Assistant and its extension with a Social Emotional Model which provides a methodology to obtain the emotional state of a group of agents. Moreover, the proposed assistant has been tested in the simulation of a retirement home that emulates real-life scenarios. The aim is to create a suggestion system that aids the social workers of a retirement

${ }_{85}$ home to plan and schedule ADLs according to the residents emotional status, promoting happiness of the group.

The rest of the paper is structured as follows, section 2 explains the related work, with related AAL projects and the technologies and concepts used; section 3 explains the Cognitive Life Assistant and its extension to get and manage emotional states; section 4

9o discusses the simulation of a retirement home and the emotional response of the agents (posing as possible users) and the validation of the actions performed by the platform; finally, section 5 presents the discussion, which details the results and creates a parallel between the simulation and a real life implementation.

\section{Related work}

Ambient Intelligence [18],[19] changed the concept of smart homes, introducing new devices and systems that help to improve people's life quality. Systems that learn 
our tastes, smart homes that help reducing energy consumption [20], safer homes for elderly [21],[22], among other applications. To achieve this, ambient intelligence systems employ different artificial intelligence tools, sensor networks, mobile Internet connections and new and sophisticated embedded devices. AmI applications imagine a future where technology surrounds users [23], and help them in their daily lives. Nevertheless, we can detect a lack of research on how to use the existing technology to the best possible effect. The automatic recognition of human activities, mainly human emotions, in which human base a significant part of their decisions, is an 105 obvious prerequisite for new AmI applications, and requires novel methods to improve recognition rates, enhancing automatic decision making, and preserving the privacy of monitored individuals. These are challenging issues, which must be addressed in current and future AmI applications.

In this section, we first analyze the Cognitive Assistants concept and the Emotional models, serving as a stepping stone for the analysis of some previous works in the area of AmI; they emphasize the importance of a strong relationship between users and the system. After this, some works which includes the emotional states area are introduced, showing how emotions can be used to a better representation of user's behaviors.

\subsection{Cognitive Assistants}

In the latest years the theme of Cognitive Assistants (CA) has been developed to evolve the core values of the AAL, translating them into usable technological platforms [24, 25, 26]. The CA's evolved from the Cognitive Orthotics area into interoperational platforms, mostly directed to elderly users, that focuses in actions that change the everyday activities, such as remembering of medication, monitoring of falls or reading

${ }_{120}$ biosignals, among others, with the goal of helping cognitively and physically. The technological goal is to be as heterogeneous as possible, meaning that any and every sensor, actuator and service (software or not) can be connected to the platform and communicate with the rest of the platform components. A CA can range from a game that stimulates the memory and the cognitive response to a complete system that has

${ }_{125}$ sensors that communicate their status to a central system, actuators that change the elements in the environment according to the response of the central system, and reports these actions to an external service provider.

There are implementations of CA's done in the past, some are services, like the Jogger [27] that resorts to remind users of items or events that happened, to reinforce 130 their memories; the CASAS project [28] monitors a subset of ADL tasks to identify consistency and completeness in daily activities; and the Autominder [29] that provides users with reminders averaging between what the users are doing and what should be doing; and some are robotic systems such as Robovie [30], Autom [31], and Paro [32] that serve as interacting interfaces. Nevertheless, there are several issues with these 135 projects, such as lack of interaction with the users, high level of failures in detecting activities, poor personalization, and cost, among others.

To achieve the better results, personalization is the key element, providing a humanlike feel to the operation process, meaning that the usual procedure of "one-size-fitsall" is really not appropriate to most of the users as they fail to attend to each user conditionings and cognitive status, which in these type of problems is imperative that 
they are addressed to guarantee that the proposed solution is well adopted by each user [33].

A pioneer advance is the usage of human emotions to model the services and environments [34]. In our point of view emotions play a key role in the development of personalized solutions since emotions reflect the state that the person is and therefore, each response can be adjusted not only to that person as well as his/her emotional status (joy, sadness, etc.).

\subsection{Emotion-based works}

Human emotional states are defined as the way humans can express how they feel in a specific period of time. These emotions can be affected by a great number of internal or external factors. Some examples of internal factors are the personality and the cognitive processes such as attention. On the other hand, external factors are mainly related with the environment, relationships among persons and cultural expectations [35]. These factors involve many aspects of our lives, determining how people behave in different situations or being the responsible of people's response to certain stimuli. These responses are governed by the personality. Based on this, human emotions are critical in any computational system which tries to simulate human behaviors. These simulation processes need to incorporate different computational models that allow us to give personality and/or emotions to artificial intelligent entities. Over the last few ${ }_{160}$ years different approaches have been developed which deepen in this idea. We can find two relevant emotional models, the OCC Model (i.e. [36]) presented by Ortony, Clore \& Collins and the PAD model presented by Albert Mehrabian and James A. Russell[37]. These models are frequently used in applications where an emotional state can be simulated. The OCC model provides a good starting point to integrate an emotional model into an intelligent software entity. However, the $O C C$ model presents one important design problem due to its high dimensionality. In this sense, the $P A D$ ( $P=$ Pleasure, $A=$ Arousal and $D=$ Dominance) model is a simplification of the $O C C$ model. This model allows the representation of the emotion in a $\mathbb{R}^{3}$ space. Each one of the components conforming this emotional model, allows to represent a measure of a 17 emotional state. According to this, the model obtains a numerical representation of all the emotions [38].

One of the existing works tries to emulate emotions in virtual humans [39]. This work, called WASABI ([W]ASABI [A]ffect [S]imulation for [A]gents with [B]elievable [I]nteractivity), tries to achieve the simulation of primary and secondary emotions using 175 virtual human's cognitive reasoning capabilities combined with simulated embodiment. The simulation and direct expression of primary emotions is based on the idea of capturing an agent's bodily feeling as a continuous progression in three-dimensional emotion space (PAD), while secondary emotions are understood as a type of emotions that require higher cognitive reasoning abilities and a certain sense of time. The work includes an empirical study which is reduced to three emotions, although authors believe that other emotions can be included in the validation. Another interesting work is the work done by Jain and Kobti [40]. This work simulates the response of intelligent agents in stress situations. Concretely, it includes an interesting example based on a hospital simulation system. The proposed example employs a basic hospital model where nurse 185 servicing patients interact in various static and dynamic emotional scenarios. According 
to authors, the obtained results allow to demonstrate that an increase in emotional stress leads to higher error rates in the task performance of nurses. This work, although interesting, lacks of some improvements like learning or adaptation capabilities of the involved agents.

Apart from the use of some specific emotional model, other works have tried to introduce the contagion effect that humans can feel in multiple situations. One of these works is the emotional contagion spiral model [41]. This model tries to give a solution to the emotional propagation, distinguishing among different factors that influence in the emotional contagion. This model is based on a emotional model that was proposed by

195 Barsade [42], which includes six hypotheses about how is produced the propagation of emotions. This work is applied in an evacuation simulation scenario, taking into account how human behaviors are affected by the dynamicity and propagation of emotions. Nevertheless, the complexity of these analysis provoques that these approaches are limited to only one emotion, in this case fear. So, behaviors of simulated agents are also affected by only one emotion.

Over the last few years different approaches have tried to employ emotional models to improve AAL services. AAL sevices rely on sensory data collected from the environment to reasoning about it in order to arrive at a correct diagnosis and advice or assist the users accordingly. In this sense, existing approaches try to represent human moods 205 in order to enhance users' comfort or simplify the human/systems interactions. In [43] authors propose an emotion-aware AmI architecture which evaluates human emotion experiences providing people with proper emotional services. In this approach is the user who directly communicates his mood by means of a mobile device. Other works have try to integrate pattern recognition techniques in order to automatically detect the emotional states of the people in an AAL environment. This is the case of [44] where an agent-based system aims the detection of user emotions for making recommendations for the visitors of a museum. In this case, the emotional states are not always detected and in some occasions the detected emotions are limited to a very narrowed set of basic emotions.

We want to emphasize that working with emotional states is not only to recognize or to emulate human emotions. Also, it is necessary to employ personality models providing a way to include personality to artificial intelligent entities. In this sense, the most popular personality model is the Big Five Model (OCEAN) [45]. This personality model represents and organizes the personality in five dimensions in a hierarchical way.

220 The five basic dimensions are: Openness, Conscientiousness, Extraversion, Agreeableness and Neuroticism. The OCEAN model has been used in some multi-agent systems approaches and, in our opinion, it must be basic in any emotional-based system for an AmI application.

Finally, in these types of systems the acceptance problematic exists and it should be considered when deploying the developed platforms with real people. It gives way to the following question: "If no one uses a tool it does it matter?" Thus, this issue must be considered as early as in the design process of the platform. [46, 47, 48] present an extensive overview of this problematic and present some solutions to address this issue. 


\section{Cognitive Life Assistant}

230

$$
\text { like }
$$
presented after the activity is performed or at the end of the day (showing one form to each activity sequentially). There is no active detection of the ending of the activity. To show the form we calculate the mean time needed to perform the activity and a margin 270 (10-20 minutes) and activate the form afterwards.

- Intra-operative API resorting to REST architecture, allowing the communication between the external services and the CLA.

- Flexible user interface built for the Android OS and the Web. Comprehensible information about all the care-receivers in one page.

- Intelligent scheduling system that receives, processes and notifies the users about incoming events from other users.

- Playful events scheduler. It comprehensibly schedules activities on the user free time to promote active aging.

- Sensor systems support through the interoperability features and agents incorporation.

CLA provides a modular connection system that allows two ways of adding new agents to the platform: internal and external. The internal agents are directly deployed on CLA and have to follow the internal communication protocols, benefitting from the full access to the internal data and being of faster execution time. The external agents have limited access to the CLA and the data they can receive is limited to the external interface protocol. Using a sensor platform as an example, it could be implemented as an internal or external agent, it would depend if the producer would be able and willing to comply with CLA's communication protocols and build the appropriate ontology for that agent; if the technology is incompatible with CLA or there are proprietary barriers then the agent can be connected externally, thus promoting the interoperability.

In terms of the playful events scheduler, its aim is to create an inclusive environment. This agent looks for free time in the calendar and schedules activities that the user likes. These activities are present in a database and are qualified according the user preferences; the activities can be shared with other users or be done individually. Promoting active aging means maintaining social connections and promoting social activities, preferably involving social building exercises.

The visual interface of the CLA is used to present the information about the events and activities and to collect the opinion of the users about the events and activities proposed. The collection of opinions process is constituted by a visual form that shows like and dislike buttons that the user can select. The visual form can be configured to be 
Next subsection introduces how the CLA have been extended including a social emotional model in order to emulate and work with human emotions in the decisionmaking of the assistant.

\subsection{Emotion-based CLA} through the creation of a safe environment that reflects the elderly taste. The retirement homes dedicate a large period of time to social activities that promote bonding of their users. Each activity typically occurs from 5 to 6 times on a week, having a duration of 3 to 6 hours per event. Therefore, this means that most of the elderly wake time dissatisfaction of those who do not like these activities. The group happiness decreases if there is a reasonable number of people that do not like those activities. In addition, people are quickly tired of repeating activities, resulting in a feeling of unhappiness. The activities are previously planned by a caregiver accounting the retirement home resources and the users, but the caregiver may not really know the user's preferences or fail to understand the user's emotional response to each activity.

Our aim is to take into account the user's emotional response to each activity and plan the next activity to maximize the happiness levels of all the group of people. This is achieved by perceiving each user response to a set of activities chosen randomly and their emotional response and changing the activity list to contain only the activities that triggered a positive emotional response of the group. The social emotion presented by Rincon et al. [51], provides a methodology to get the emotion of a group of agents based on the $P A D$ emotional model.

This model represents the social emotion of a heterogeneous group of entities capable of expressing and/or communicating emotions. To define a social emotional model, it is necessary to first define the representation of an emotional state of an agent according to the $P A D$ model. The emotion of an agent $a g_{i}$ is defined by a vector in a $\mathbb{R}^{3}$ space, represented by the three components that make up the $P A D$ emotional model $(P=$ Pleasure,$A=$ Arousal and $D=$ Dominance $)$. The variation of each component modifies the emotional state of the agent.

$$
\vec{E} a g_{i}=P_{i}, A_{i}, D_{i}
$$

From this emotional representation of each agent, a social emotional representation is defined as a way to represent the emotion of a group of heterogenous agents. This social emotion is composed by a triplet. This triplet consists of three vectors $(\overrightarrow{C E} A g, \vec{m} A g, \vec{\sigma} A g) . \overrightarrow{C E} A g$ is the Central Emotion $(C E)$ obtained by averaging the $P$, 305 $A, D$ values of all the agents; $\vec{m} A g$ is the Max Distance which is a vector where each component is calculated by means of the Euclidean distance of each value of the $P A D$ and the $C E$; and $\vec{\sigma} A g$ is the Standard Deviation which allows to calculate the level of emotional dispersion of a group of agents around the $C E$. A detailed explanation of this model can be find in [51].

Another important feature of this model is the possibility of calculating the emotional distances among different groups of agents or between a group of agents and a target emotion. The target emotion is dependent of the scenery, it is possible to define target 
emotions that makes the agents happy or angry. Being our model based on PAD emotional model, the target emotion is defined over the same terms. But to select a target emotion (in the PAD) there is only the necessity of knowing the final emotion. This procedure has been studied by other authors, that have created a set of tables for each emotion, using these tables we can define the different targets emotions.

This approach can be used as a feedback in the decision making process of the CLA, allowing the shift of the social emotion to a particular area of the $P A D$ space; or shifting

320 the emotional state of a group of agents in relation to other groups of agents.

Following the model defined in [34, 52], the CLA has been extended introducing a new agent called SEtA (Social Emotional Agent) that allows us to calculate the Social Emotion ( $S E)$ and, also, the distance between the $S E$ and possible target emotions. The aim of this agent is to get the emotion of all the agents of the group, and using this

325 nformation the agent is capable of calculating the social emotion of the group.

The interaction process between the CLA and the new agent can be described in the following way: In the beginning of each decision-making process, the CLA asks SEtA to calculate the social emotion $(S E)$ for the group of agents and the distance between this $S E$ and the target emotion (the target emotion is the emotion that the CLA wants ${ }_{330}$ to achieve for the group); then, the CLA, taking into account these values, decides and sends different activities to all the agents; once all agents have evaluated the activities, they send the emotional results to the SEtA in order to calculate again the SE.

The CLA is able to manage the suggestion system and schedule the shared activities on all of the participant's calendars. The module that manages this feature will have to receive the input of each user about their feeling towards each finished activity; the activities will be classified according to the group emotional response. The objective is to increase the probability of the preferred activities being chosen for future iterations. The input of the system and the user is saved on CLA databases, relating the activity with an emotion and personal response. Thereon, the activities are requalified according to those values, thus in the following activities retrieval these are used in a correlating algorithm that adjusts the events to the emotion (trying to enforce positive emotions by reinforcing them or reversing negative emotions).

Next section describes the implementation and evaluation of a case study based on this emotion-based CLA.

\section{Validation of the proposal: a retirement home simulation}

The presented proposal has been validated through a simulation of a retirement home. Specifically, the case study employs real data collected by a Portuguese institution called Fundação Manuel Francisco Clérigd ${ }^{1}$. The data used to our validation process went through the OCEAN test to determine the personality of each person. The data obtained was used to model each person as an agent. And, at the same time, this data was used to calculate the initial emotion using the Equation 2

\footnotetext{
${ }^{1}$ http://www.fundacaoclerigo.com/
} 


\begin{tabular}{|l|l|l|l|}
\hline Agent id & Pleasure & Arousal & Dominance \\
\hline Agent 0 & 0.444 & 0.049 & 0.337 \\
\hline Agent 1 & 0.6289 & 0.0093 & 0.399 \\
\hline Agent 2 & 0.3241 & -0.3217 & 0.1789 \\
\hline Agent 3 & 0.3293 & -0.0465 & 0.0613 \\
\hline Agent 4 & 0.2831 & -0.4496 & 0.4533 \\
\hline Agent 5 & 0.3091 & -0.1296 & 0.3238 \\
\hline Agent 6 & 0.5204 & -0.0080 & 0.3128 \\
\hline Agent 7 & 0.5638 & 0.0093 & 0.1798 \\
\hline Agent 8 & 0.3103 & -0.4316 & 0.3543 \\
\hline Agent 9 & 0.6560 & 0.1153 & -0.0146 \\
\hline Agent 10 & 0.2873 & -0.4906 & 0.1833 \\
\hline Agent 11 & 0.3794 & 0.2289 & 0.2069 \\
\hline Agent 12 & 0.3088 & -0.0377 & 0.0877 \\
\hline Agent 13 & 0.1070 & -0.0416 & 0.0389 \\
\hline Agent 14 & 0.86431 & -0.1456 & 0.5213 \\
\hline Agent 15 & 0.5598 & 0.0093 & -0.0142 \\
\hline Agent 16 & 0.5584 & -0.0960 & 0.4138 \\
\hline Agent 17 & 0.6161 & -0.0036 & -0.1379 \\
\hline Agent 18 & 0.6550 & -0.2007 & -0.0820 \\
\hline Agent 19 & 0.2031 & -0.2866 & 0.3234 \\
\hline
\end{tabular}

Table 1: PAD initial values for the 20 agents used in the simulation scenarios.

\begin{tabular}{|l|l|}
\hline $\overrightarrow{C E} P, A, D$ & {$[0,4452105-0,1249450,20634]$} \\
\hline$\vec{\sigma} P, A, D$ & {$[0,4907634560,2209379820,717962146]$} \\
\hline$\vec{m} P, A, D$ & {$[0,79290,49061,038]$} \\
\hline
\end{tabular}

Table 2: Initial social emotion of the group of agents used in the different scenarios.

$$
\begin{array}{r}
P_{0}=0.59 * A+0.19 * N+0.21 * E \\
A_{0}=-0.59 * N+0.30 * O+0.15 * O \\
D_{0}=0.60 * E-0.32 * A+0.25 * O+0.17 * C
\end{array}
$$

The values obtained using the Equation 2 were applied to the agents as the initial emotion. Based on this information the caregiver agent knows what is the initial emotional state. At this center, there is a caregiver agent in charge of sending different tasks with the goal of producing an emotion to all the individuals within the retirement home as close as possible to happiness. There are two types of tasks: Inside and Outside Activities. Each one of the individuals is represented by an agent, which has an emotional response according to the tasks it receives. Moreover, as the individual emotions of the agents change, the social emotion of the whole retirement home will 360 change accordingly [51].

The different scenarios have been designed in order to show how the social emotion 
facilitates the decision making of the CLA. All these scenarios are composed by 20 agents representing the elderly people at the retirement home that receive the activities from the caregiver agent. These agents have at the beginning of the execution of each scenario the initial emotions shown in Table 1, each one of these emotions was obtained by the OCEAN model for each one of such agents.

These initial emotions are needed to start the system and obtain a first social emotion of the group. Once obtained this first vision of the emotion of the group, the caregiver agent sends activities for each agent to try to change the group emotion through the 370 different activities. Each agent responds differently to each activity, affecting to its emotional state. The emotional change is made using a fuzzy logic algorithm following a similar approach of the work made in [53]. This algorithm has as input the activity that was sent by the caregiver agent. Inside of each fuzzy logic function we can find a series of rules, each one reacting in a different way to the activity. These rules allow to specify if an agent prefers more an activity than other. The response given by the fuzzy logic corresponds to an emotion expressed in PAD values associated to this specific activity. All of such $P A D$ values are then used to calculate the social emotion [51] of the group of agents. Figure 1 shows an example of the fuzzy logic functions employed in this process.



Figure 1: Example of the fuzzy logic function employed in the emotion recognition of each PAD value.

In each one of the different simulation presented in this section, the aim is to reach a target emotion that in these cases is happiness. To try to achieve this goal, the social emotional model has two measures (dispersion and maximum distance) that allow us to know if we are reaching the target. The dispersion and maximum distance of such initial values with respect to the target emotion we have taken into account in the simulation Happy are shown in Table 2. Once the activity has ended, each agent sends its emotional state to the SEtA agent that evaluates the social emotion of the group and the distance between this current social emotion and the goal emotion, that is, global happiness. So, the caregiver agent's purpose is to minimize such distance using the different tasks available to it. Figure 2 shows the schema of the communication process between all 


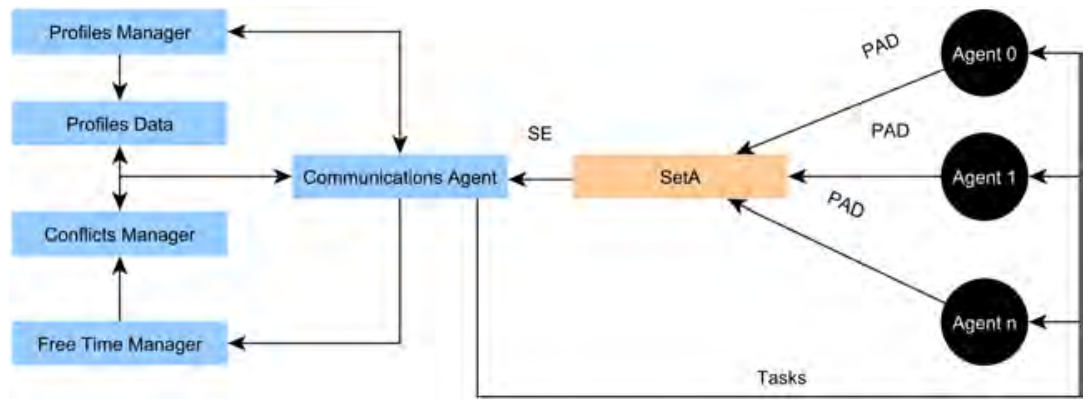

Figure 2: Simulation execution schema of the extended CLA.

390

entities that composed the simulation. These entities can be divided into two groups:

- CLA: (in blue) These are the agents that represent the CLA platform. It has the task of sending the different activities to the agents. These activities allow to modify the emotional state of each agent.

- SetA Agent: (in brown) it has the task of calculating the social emotion of the group. This agent receives PAD vectors corresponding to the emotional state of each agent. Using this information the SetA agent calculates the social emotion. This social emotion is sent to the caregiver agent to decide about changing the activities or not.

The operation of the system relies on the CLA as a pivoting elements that connects all parts of the system. It is responsible of sending the activities to the agents (Agent 1, ..., Agent $\mathrm{n}$ ) and collecting the information from the SetA agent about the social emotion and selecting new events according to that information.

In the next subsections we have developed different scenarios changing the fuzzy logic function that implements the emotional response of the different agents. So we have five different scenarios: the first one corresponds to functions that are prone to like the activities proposed by the caregiver agent, the second one corresponds to functions that dislike the activities (or the repetition of them), the third one corresponds to five agents with functions that tend to like the proposals and fifteen to dislike them, the fourth one corresponds to fifteen agents that tend to be bored by the caregiver proposals 410 and five agents that tend to be happy, and the last one corresponds to half the agents being happy and half being bored with the proposals. For each scenario 30 different executions have been carried out. The different figures presented in each scenario show for each datapoint the average value of the set of executions.

\subsection{Scenario 1: All Agents like the activities}

415 The first case analyzed is one in which the fuzzy logic functions used for the emotional response of the agent lead them to get close to Happy. If the agents like the tasks provided by the caregiver agent they will be happy. However, each agent have the possibility of reducing its emotional state (tending to be more bored) if the activity is 
repeated for a long time or if they dislike the activity. This emotional reaction causes a change in the agent emotion from happy to boring. A graphical representation of this example can be seen in Figure 3 showing the emotional difference, which is calculated between the social emotion and the target emotion emphHappy. In this case all agents tend to like the activities sent by the caregiver agent and therefore the difference between their social emotion and the target emotion (Happy) is decreased till in the end it is very 425 low.

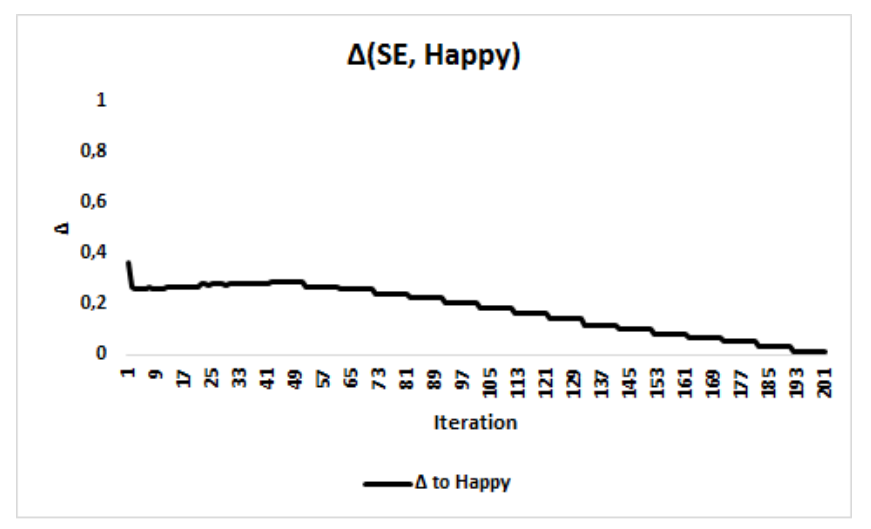

Figure 3: Scenario 1: All Agents are Happy.

Figure 4 represents the evolution of the dispersion around the group of agents' social emotion in this first scenario. These dispersions are calculated for each component of the $P A D$ vector. It can be observed that they become lower as time passes, meaning that this is a very cohesive group that receives very positively all the activities suggested by the caregiver agent.

The maximum distance measurement lets us know if there is any agent far away from the target emotion. To obtain this measure we use the Euclidean distance between two points in the space. This distance is different from the maximum distances of the model of social emotion, because these are the maximum distances for each component of the vector $P A D$. A graphical representation of this example can be seen in Figure 5 showing the max distance variation: as all agents are happy the max distance is low. As agents modify their emotions depending on the task repetition, the graph shows some variation in the max distance. These variations indicate that the agent has received from the caregiver recurring activities, making the maximum distance to increase.

${ }_{440}$ To sum up, this scenario corresponds to a very cohesive group of people that responds to the activities suggested by the caregiver agent very positively reaching a happy emotion as intended.

\subsection{Scenario 2: All Agents are Bored by the activities}

The second case analyzed is one in which the fuzzy logic functions controlling the 445 emotional response of the agents are not responding properly to the suggestions of the caregiver agent, so the agents tend to get bored. Moreover, there is a possibility of 


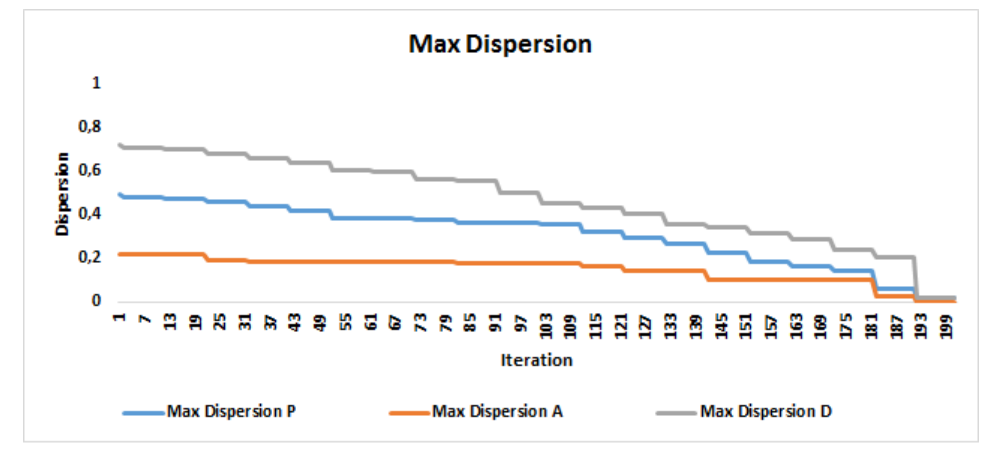

Figure 4: Scenario 1: Max dispersion between the emotion of each agent.

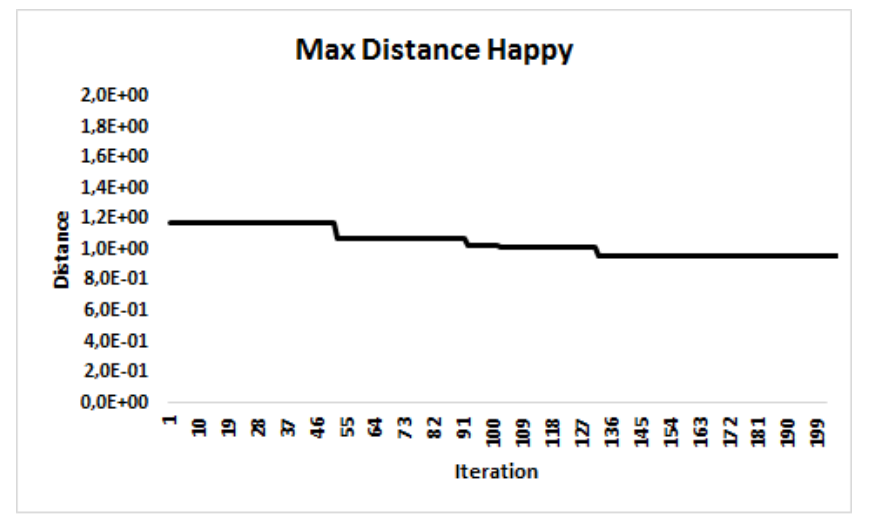

Figure 5: Scenario 1: Max distance between happy emotion and the emotion of each agent.

getting bored if the activity is repeated by a long time. This emotional reaction causes a change in the agent emotion from happy to boring. A graphical representation of this example can be seen in Figure 6 showing the emotional difference, which is calculated between the social emotion and the target emotion (happy).

The progression of the graph (the high values) are a result of the difference between the emotions happy and bored, thus the high values show that the agents are far way from the target emotion happy.

Figure 7 represents the evolution of the dispersion around the group of agents' social emotion in this second scenario. These dispersions are calculated for each component of the $P A D$ vector. It can be observed that they become greater as time passes, meaning that the group is getting disjointed as it receives the activities suggested by the caregiver agent.

A graphical representation of this example can be seen in Figure 8 showing the max 460 distance variation obtained in the group of agents. That is, as all agents are getting bored very quickly, the max distance is achieved at the same rate. 




Figure 6: Scenario 2: All Agents are Bored.

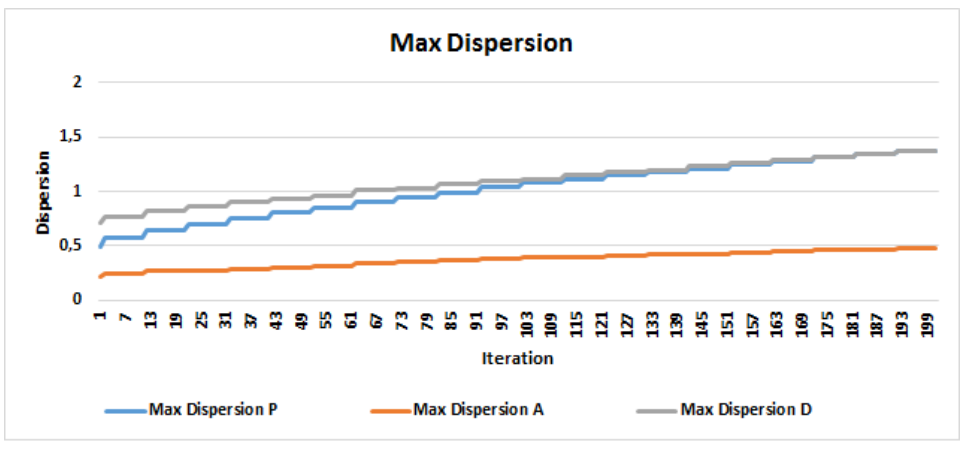

Figure 7: Scenario 2: Max dispersion between the emotion of each agent.

\subsection{Scenario 3: Five Agents are Happy and Fifteen Agents are Bored with the activities}

The fuzzy logic functions used in this scenario, makes the agents to be divided into two groups: a first group composed by five agents, and the other by fifteen agents. A

465 graphical representation of this example can be seen in Figure 9 showing the evolution of the emotional difference between the social emotion and the target emotion. At this Figure, it can be observed as the social emotion tends to be less happy than in the scenario 1.

Figure 10 represents the evolution of the dispersion around the group of agents' social emotion in this third scenario. These dispersions are calculated for each component of the $P A D$ vector. It can be observed that they become greater as time passes, meaning that the group is getting disjointed as it receives the activities suggested by the caregiver agent. This behavior makes sense as five agents get happy with such activities but fifteen get bored with them.

A graphical representation of this example can be seen in Figure 11 showing the max 


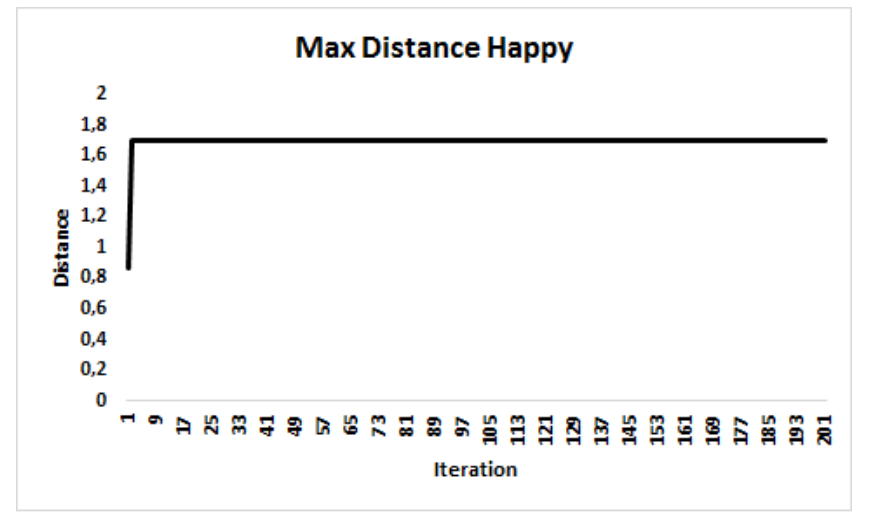

Figure 8: Scenario 2: Max distance between target emotion happy and the emotion of each agent.

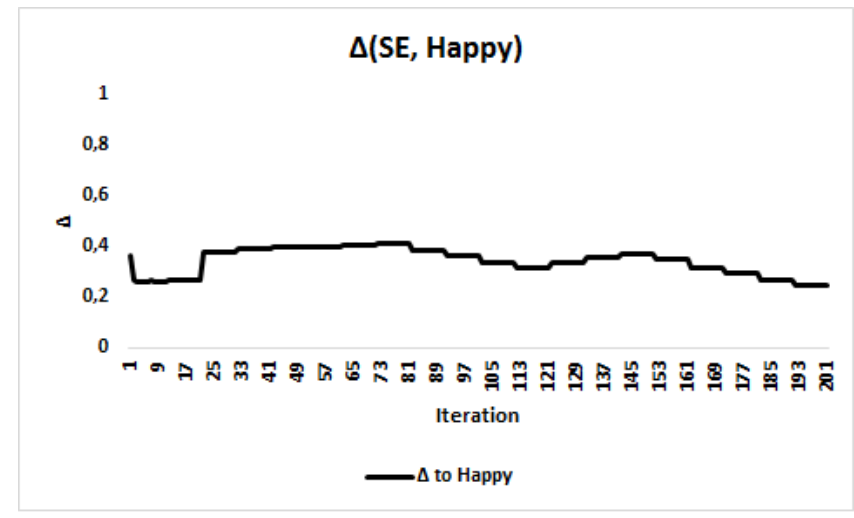

Figure 9: Scenario 3: Five Agents Happy and Fifteen Agents Bored.

distance variation. As there exist agents getting bored very quickly, and maintaining such emotion during the scenario execution, the max distance achieves and maintains high values very soon.

\subsection{Scenario 4: Fifteen Agents are Happy and Five Agents are Bored with the activities}

In this scenario we have situation contrary to the one presented in the previous one. A graphical representation of this example can be seen in Figure 12 showing the evolution of the emotional difference. It can be observed there that the difference get close to happy, although not very close due to the influence of the five agents that tend to get bored.

485 Figure 13 represents the evolution of the dispersion around the group of agents' social emotion in this fourth scenario. These dispersions are calculated for each component of the $P A D$ vector. It can be observed that they maintains more or less similar 


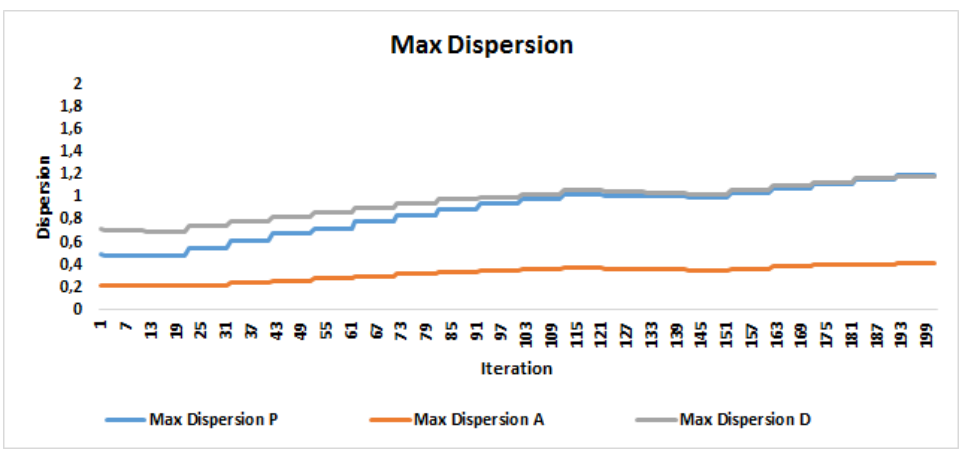

Figure 10: Scenario 3: Max dispersion between the emotion of each agent.

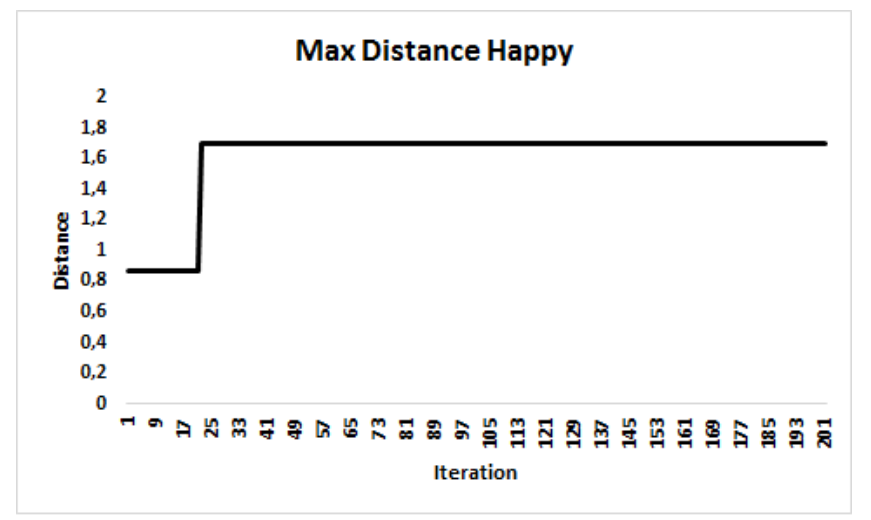

Figure 11: Scenario 3: Max distance between target emotion happy and the emotion of each agent.

values during all the scenario execution excepting the pleasure component that increases its dispersion.

490 A graphical representation of this example can be seen in Figure 14 showing the max distance variation. It can be observed that there will exist at least some agent that will reach and maintain the bored emotion, but as commented in the previous figures, the group social emotion tends to happy.

\subsection{Scenario 5: Ten Agents are Happy and Ten Agents are Bored with the activities}

495 The fuzzy logic functions used in this scenario, makes the agents to be divided into two groups of ten agents. A graphical representation of this example can be seen in Figure 15 showing the evolution of the emotional difference. As time passes we can observe a slight increasing because of the effect of repetition in the caregiver suggestions.

500 A graphical representation of this example can be seen in Figure 16 showing the max distance variation. It can be observed that there will exist at least some agent that 


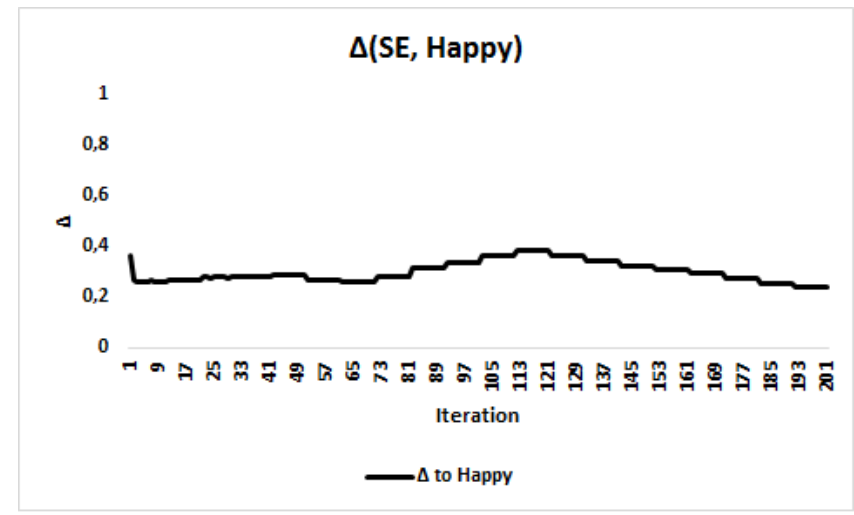

Figure 12: Scenario 4: Fifteen Agents Happy and Five Agents Bored.

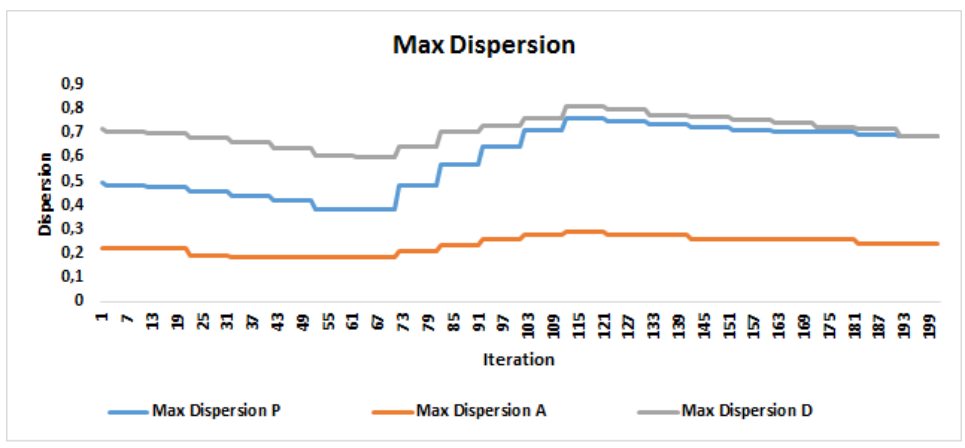

Figure 13: Scenario 4: Max dispersion between the emotion of each agent.

will reach and maintain the bored emotion.

A graphical representation of this example can be seen in Figure 17 showing the max distance variation. As all agents are happy the max distance is low. As the agent can modify their emotion depending on the task repetition, the graph shows some variation in the max distance. These variations indicate that the agent has sent caregiver recurring activities, making the maximum distance increase.

Table 3 shows the final values of the variables measured in each scenario. It shows the distances to Happy and to Bored and their position in the PAD. While in scenario 5101 and 2 the results are straightforward, the scenario 3 and 4 present interesting results. In the scenario 3 its observable the high values of $\Delta_{\text {Happy }}$ and $\Delta_{\text {Bored }}$, this is explained because the two groups display the opposite emotions very quickly, thus a balance is established quickly, as observed in Figure 10. The dispersion dos not evolve significantly, the agents clump into two groups and maintain that groups throughout the execution, ${ }_{515}$ thus the almost equilibrium between the two emotions. The scenario 4 is the inversion of the scenario 3 , therefore, the behaviour of the agents are alike the one showed in 


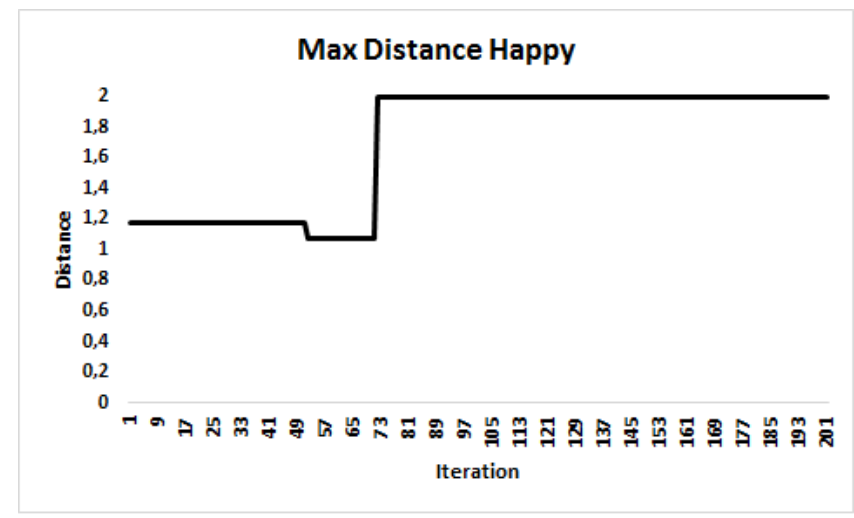

Figure 14: Scenario 4: Max distance between target emotion happy and the emotion of each agent.

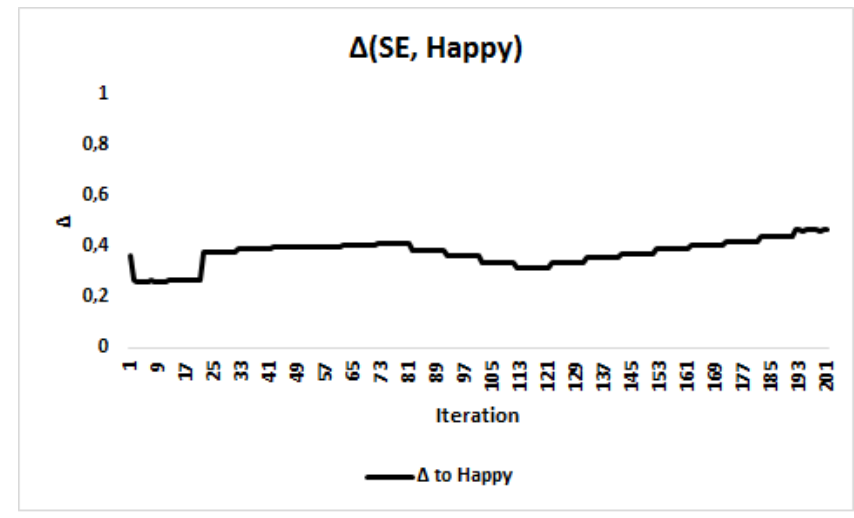

Figure 15: Scenario 5: Ten Agents Happy and Ten Agents Bored.

scenario 3 but inverted.

\begin{tabular}{|l|l|l|l|l|l|}
\hline & Scenario 1 & Scenario 2 & Scenario 3 & Scenario 4 & Scenario 5 \\
\hline$\Delta_{\text {Happy }}$ & 0,237 & 0,512 & 0,574 & 0,584 & 0,398 \\
\hline$\Delta_{\text {Bored }}$ & 0,598 & 0,305 & 0,602 & 0,617 & 0,421 \\
\hline$\vec{\sigma} P, A, D$ Happy & {$[0,3740,1810,564]$} & {$[1,3120,4611,321]$} & {$[0,7020,2570,725]$} & {$[0,6890,2380,715]$} & {$[0,9850,3501,010]$} \\
\hline$\vec{\sigma} P, A, D$ Bored & {$[1,5290,6191,209]$} & {$[0,1740,1450,139]$} & {$[1,3270,5411,049]$} & {$[1,3270,5411,049]$} & {$[1,0880,4500,860]$} \\
\hline$\vec{m} P, A, D$ Happy & {$[0,6960,44971,038]$} & {$[1,3750,4751,37]$} & {$[1,3750,4751,37]$} & {$[1,3750,4751,37]$} & {$[1,3750,4751,37]$} \\
\hline$\vec{m} P, A, D$ Bored & {$[0,6191,2092,045]$} & {$[1,3210,1750,145]$} & {$[1,52940,61991,209]$} & {$[1,2092,0451,327]$} & {$[0,61991,2122,045]$} \\
\hline
\end{tabular}

Table 3: Data Representing the final situation of the Five Scenarios. 


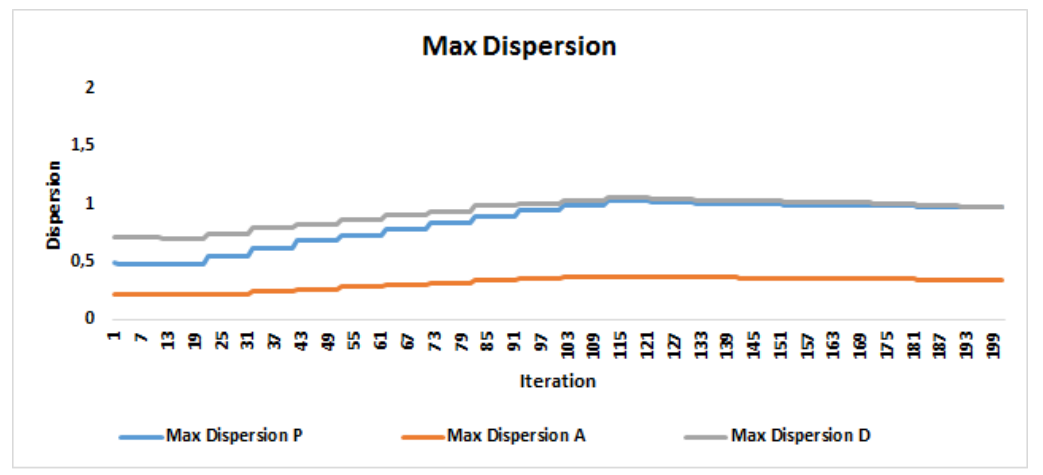

Figure 16: Scenario 5: Max dispersion between the emotion of each agent.

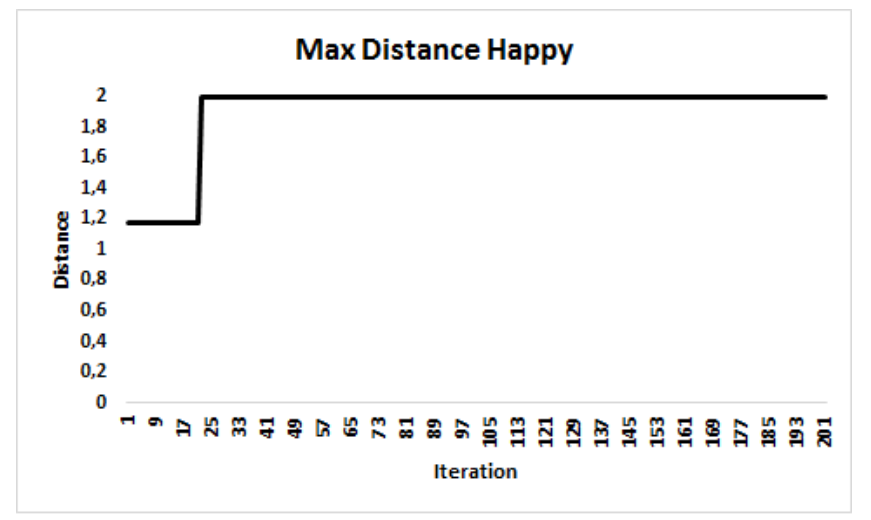

Figure 17: Scenario 5: Max distance between target emotion happy and the emotion of each agent.

\section{Discussion}

This paper deepens in the use of emotions in an AAL application, as a way to improve the decision making process in this kind of systems. Concretely, the proposal consists of the implementation and tests of a ludic events scheduler aimed to elderly people that are resident on retirement homes to promote active aging through the daily proposal of activities that people generally like.

The proposed AAL system has been tested through the implementation of an agentbased simulation environment where different scenarios have been designed and analysed. Results show how the inclusion of emotions and the psychological state of elderly people in the senior centre allows a more accurate activity scheduler. The proposed activities can be measured and analysed according to the emotional changes in the group, allowing the detection of undesirable situations.

${ }_{530}$ The simulation results show interesting and positive results, where the system had in all of the cases had an impact in all scenarios (unfortunately not all were positive as 
expected). From them two conclusions can be derived:

- The simulated users tend to follow the community more than the positive effects of the suggestions;

- The simulated users react rapidly to changes and go to emotional extremes quickly.

The simulation then can be used as a platform for pre-implementation in real scenarios, as it presents the most extreme outcome, thus highlighting possible social implications on those outcomes. Unfortunately, there is not much research in this area, and due to the specification of the platform and the proposed scenarios we are unable to provide

${ }_{540}$ a scientific comparison to other systems. As explained in section 2, there are projects that aim the care for the elderly, and others in terms of detecting the emotional state of the human-beings, but none that relates them, having as goal the direct interaction and establishing a human-machine platform.

Due to external conditions (data protection liabilities and privacy concerns) we were unable to implement this platform on a real environment; thus, we are unable to provide results of a real application. Until that is the case we will dedicate to the development and fine-tuning of the platform, considering new implementations, or in an extreme situation, address other type of users.

As future work, we want to measure the effect over the human behaviours produced 550 by possible emotional propagations among the people involved in the senior centre, including elderly and monitors. To do this a dynamic emotional model which considers aspects like affinity, empathy and emotional attraction must be integrated in the proposed simulation environment. Moreover, the acceptance problematic will be considered, to establish an operation guideline, that tackles possible problems that the users may ${ }_{555}$ present, and increase their acceptance values. Thus, effectively improving how elderly people react to new technologies and accept that they give them suggestions and change their daily living.

\section{Acknowledgements}

Angelo Costa thanks the Fundação para a Ciência e a Tecnologia (FCT) the Post-Doc scholarship with the Ref. SFRH/BPD/102696/2014. This work is also supported by COMPETE: POCI-01-0145-FEDER-007043 and FCT - Fundação para a Ciência e Tecnologia within the Project Scope: UID/CEC/00319/2013 and partially supported by the MINECO/FEDER TIN2015-65515-C4-1-R and the FPI grant AP2013-01276 awarded to Jaime-Andres Rincon.

\section{References}

[1] L. D. Harris-Kojetin, M. Sengupta, E. Park-Lee, R. Valverde, Long-Term Care Services in the United States: 2013 Overview, Tech. rep., National Center for Health Statistics (2013).

[2] Long-Term Care Financing Project, Who needs long-term care?, Tech. rep., Georgetown University (2003). URL https://goo.gl/Nkpp3B 
[3] AARP, Beyond 50.03: A Report to the Nation on Independent Living and Disability, Tech. rep., AARP (2003).

URL http://assets.aarp.org/rgcenter/il/beyond_50_il_1.pdf

575

580

[12] J. Bravo, D. Cook, G. Riva, Ambient intelligence for health environments, Journal of Biomedical Informatics 64 (2016) 207-210.

[13] P. Klasnja, W. Pratt, Healthcare in the pocket: Mapping the space of mobile-phone health interventions, Journal of Biomedical Informatics 45 (1) (2012) 184-198.

[14] A. Fernández-Caballero, A. Martínez-Rodrigo, J. M. Pastor, J. C. Castillo, E. Lozano-Monasor, M. T. López, R. Zangróniz, J. M. Latorre, A. Fernández-Sotos, Smart environment architecture for emotion detection and regulation, Journal of Biomedical Informatics 64 (2016) 55-73.

[15] AAL4ALL, AAL4ALL - Ambient Assisted Living For All (2015). URL www.aal4all.org

[16] L. M. Camarinha-Matos, J. a. Rosas, F. Ferrada, A. I. Oliveira, BRAID Active Ageing Scenarios, Tech. rep. (2011).

URL http://goo.gl/zUdI21 
[28] P. Rashidi, D. Cook, Keeping the Resident in the Loop: Adapting the Smart Home to the User, IEEE Transactions on Systems, Man, and Cybernetics - Part A: Systems and Humans 39 (5) (2009) 949-959.

[29] M. E. Pollack, L. Brown, D. Colbry, C. E. McCarthy, C. Orosz, B. Peintner,

[17] A. Fiske, J. L. Wetherell, M. Gatz, Depression in older adults., Annual Review of Clinical Psychology 5 (2009) 363-389.

[18] M. Satyanarayanan, A catalyst for mobile and ubiquitous computing, Pervasive Computing, IEEE 1 (1) (2002) 2-5.

[19] E. Mangina, J. Carbo, J. M. Molina, Agent-based ubiquitous computing, Atlantis Press : World Scientific, Amsterdam; Paris, 2009.

[20] D.-M. Han, J.-H. Lim, Smart home energy management system using IEEE 802.15. 4 and zigbee, Consumer Electronics, IEEE Transactions on 56 (3) (2010) $1403-$ 1410 .

[21] S. S. Intille, Designing a home of the future, IEEE pervasive computing 1 (2) (2002) 76-82.

[22] M. Satyanarayanan, Pervasive computing: Vision and challenges, Personal Communications, IEEE 8 (4) (2001) 10-17.

[23] J. C. Augusto, Ambient intelligence: The confluence of ubiquitous/pervasive computing and artificial intelligence, in: D. A. J. Schuster (Ed.), Intelligent Computing Everywhere, Springer London, 2007, pp. 213-234.

[24] J. A. Jorge, Adaptive tools for the elderly: new devices to cope with age-induced cognitive disabilities, in: WUAUC'01 Proceedings of the 2001 EC/NSF workshop on Universal accessibility of ubiquitous computing: providing for the elderly, ACM, 2001, pp. 66-70.

[25] A. L. Liu, P. A. Brown, G. Borriello, M. Harniss, H. Kautz, K. Johnson, Learning User Models to Improve Wayfinding Assistance for Individuals with Cognitive Impairment, Workshop on Interactive Systems in Healthcare.

[26] A. Costa, E. Martinez-Martin, A. P. del Pobil, R. Simoes, P. Novais, Find It An Assistant Home Agent, in: Trends in Practical Applications of Agents and

635 Multiagent Systems, Vol. 221, Springer, 2013, pp. 121-128. doi:10.1007/ 978-3-319-00563-8_15.

[27] E. Kamar, E. Horvitz, Jogger: Models for Context-Sensitive Reminding, in: AAMAS ' 11 The 10th International Conference on Autonomous Agents and Multiagent Systems, The Tenth International Conference on Autonomous Agents and Multiagent Systems, 2011, short Paper.

\section{S. Ramakrishnan, I. Tsamardinos, Autominder: an intelligent cognitive orthotic} system for people with memory impairment, Robotics and Autonomous Systems 44 (3-4) (2003) 273-282. 
[30] A. M. Sabelli, T. Kanda, N. Hagita, A Conversational Robot in an Elderly Care Center: An Ethnographic Study, in: Proceedings of the 6th International Conference on Human-robot Interaction, HRI '11, ACM, New York, NY, USA, 2011, pp. $37-44$.

[31] C. D. Kidd, C. Breazeal, Robots at home: Understanding long-term human-robot interaction, in: IEEE/RSJ International Conference on Intelligent Robots and Systems, 2008. IROS 2008, 2008, pp. 3230-3235.

[32] K. Wada, T. Shibata, Robot therapy in a care house - its sociopsychological and physiological effects on the residents, in: Proceedings 2006 IEEE International Conference on Robotics and Automation, 2006. ICRA 2006, 2006, pp. 3966-3971.

[33] F. Sousa, L. Viola, L. Ferreira, G. Trevisan, D. Cunha, J. Alves, R. Simões, An ecosystem of products and systems for ambient intelligence - the AAL4ALL users perspective., Studies in health technology and informatics 177 (2012) 263-71.

[34] J. Rincon, V. Julian, C. Carrascosa, An Emotional-based Hybrid Application for Human-Agent Societies, in: SOCO 2015, Vol. 368, 2015, pp. 203-214.

[35] N. A. Fox, S. D. Calkins, The development of self-control of emotion: Intrinsic and extrinsic influences, Motivation and emotion 27 (1) (2003) 7-26.

${ }_{665}$ [36] A. Ortony, The Cognitive Structure of Emotions, Cambridge University Press, 1990.

[37] A. Mehrabian, Analysis of affiliation-related traits in terms of the PAD temperament model, The Journal of Psychology 131 (1) (1997) 101-117.

[38] R. F. Bales, Social Interaction Systems: Theory and Measurement, new edition edition Edition, Transaction Publishers, New Brunswick, NJ, 2001.

[39] C. Becker-Asano, I. Wachsmuth, Affective computing with primary and secondary emotions in a virtual human, in JAAMAS 20 (1) (2010) 32-49.

[40] D. Jain, Z. Kobti, Simulating the effect of emotional stress on task performance using OCC, in: Advances in Artificial Intelligence, Springer, 2011, pp. 204-209.

[41] T. Bosse, M. Hoogendoorn, M. C. A. Klein, J. Treur, C. N. van der Wal, A. van Wissen, Modelling collective decision making in groups and crowds: Integrating social contagion and interacting emotions, beliefs and intentions, Autonomous Agents and Multi-Agent Systems 27 (1) (2013) 52-84.

[42] S. G. Barsade, The ripple effect: Emotional contagion and its influence on group behavior, Administrative Science Quarterly 47 (4) (2002) 644-675.

[43] G. Acampora, V. Loia, A. Vitiello, Distributing emotional services in ambient intelligence through cognitive agents, Service Oriented Computing and Applications 5 (1) (2011) 17-35. 
[44] M.-A. Puică, I. Mocanu, A.-M. Florea, Intelligent Distributed Computing VII: IDC 2013, Springer International Publishing, Cham, 2014, Ch. Agent-Based System for Affective Intelligent Environment, pp. 335-342.

[45] R. J. Harvey, W. D. Murry, S. E. Markham, A "Big Five" scoring system for the Myers-Briggs type indicator, in: annual conference of the Society for Industrial and Organizational Psychology, Orlando, 1995.

[46] A. Holzinger, G. Searle, M. Wernbacher, The effect of previous exposure to technology on acceptance and its importance in usability and accessibility engineering, Universal Access in the Information Society 10 (3) (2010) 245-260. doi:10.1007/s10209-010-0212-x

[47] A. Holzinger, K. Schaupp, W. Eder-Halbedl, An investigation on acceptance of ubiquitous devices for the elderly in a geriatric hospital environment: Using the example of person tracking, in: Lecture Notes in Computer Science, Springer Berlin Heidelberg, pp. 22-29. doi:10.1007/978-3-540-70540-6_3.

[48] D. Singh, E. Merdivan, I. Psychoula, J. Kropf, S. Hanke, M. Geist, A. Holzinger, Human activity recognition using recurrent neural networks, in: Lecture Notes in

700 Computer Science, Springer International Publishing, 2017, pp. 267-274. doi: 10.1007/978-3-319-66808-6_18.

[49] Â. Costa, J. C. Castillo, P. Novais, A. Fernández-Caballero, R. Simoes, Sensordriven agenda for intelligent home care of the elderly, Expert Syst. Appl. 39 (15) (2012) 12192-12204. doi:10.1016/j.eswa.2012.04.058.

[50] A. Costa, P. Novais, R. Simoes, A caregiver support platform within the scope of an ambient assisted living ecosystem., Sensors 14 (3) (2014) 5654-5676. doi: $10.3390 / \mathrm{s} 140305654$.

[51] J. A. Rincon, V. Julian, C. Carrascosa, Social Emotional Model, in: Springer (Ed.), Advances in Practical Applications of Agents: The PAAMS Collection, no. 9086 in LNCS, 2015, pp. 199-210.

[52] J. Rincon, V. Julian, C. Carrascosa, Representing Social Emotions in MAS, in: 13th International Conference on PAAMS, Vol. 9086 of LNAI, 2015, pp. 308-311.

[53] A. Nanty, R. Gelin, Fuzzy controlled PAD emotional state of a NAO robot, in: 2013 Conference on Technologies and Applications of Artificial Intelligence (TAAI), 2013, pp. 90-96. 